\title{
Criminologie
}

\section{Victimisation : réalité préoccupante pour les jeunes pris en charge par la DPJ}

\author{
Katie Cyr, Claire Chamberland, Marie-Ève Clément et Geneviève Lessard
}

Volume 47, numéro 1, printemps 2014

La polyvictimisation des jeunes

URI : https://id.erudit.org/iderudit/1024013ar

DOI : https://doi.org/10.7202/1024013ar

Aller au sommaire du numéro

Éditeur(s)

Les Presses de l’Université de Montréal

ISSN

0316-0041 (imprimé)

1492-1367 (numérique)

Découvrir la revue

Citer cet article

Cyr, K., Chamberland, C., Clément, M.-È. \& Lessard, G. (2014). Victimisation : réalité préoccupante pour les jeunes pris en charge par la DPJ. Criminologie, 47(1), 187-211. https://doi.org/10.7202/1024013ar
Résumé de l'article

Cet article compare la violence annuellement vécue par un échantillon de jeunes (2 à 17 ans) Québécois pris en charge par la Direction de la protection de la jeunesse (DPJ) et celle subie par les jeunes de la population générale. Les résultats démontrent que les jeunes pris en charge vivent significativement plus de violence et qu'une grande partie d'entre eux font face à de la violence chronique dans plusieurs sphères de leur vie. Les trajectoires susceptibles d'expliquer leur risque accru de victimisation et les implications pour l'intervention sont discutées. Les besoins multiples de ces jeunes soulignent l'importance de développer une vision plus holistique de leur vécu de victimisation et de leurs problèmes. Une plus grande collaboration des divers intervenants et organismes impliqués auprès des jeunes est susceptible de favoriser une approche moins fragmentée et une intervention mieux adaptée. 


\title{
Victimisation: réalité préoccupante pour les jeunes pris en charge par la DPJ
}

\author{
Katie Cyr ${ }^{1}$ \\ Professeure associée \\ Université de Montréal \\ Katie.cyr@umontreal.ca \\ Claire Chamberland \\ Professeure honoraire \\ Université de Montréal \\ Claire.chamberland@umontreal.ca \\ Marie-Ève Clément \\ Professeure titulaire \\ Université du Québec en Outaouais \\ marie-eve.clement@uqo.ca \\ Geneviève Lessard \\ Professeure titulaire \\ Université Laval \\ genevieve.lessard@svs.ulaval.ca
}

RÉSUMÉ - Cet article compare la violence annuellement vécue par un échantillon de jeunes (2 à 17 ans) Québécois pris en charge par la Direction de la protection de la jeunesse (DPJ) et celle subie par les jeunes de la population générale. Les résultats démontrent que les jeunes pris en charge vivent significativement plus de violence et qu'une grande partie d'entre eux font face à de la violence chronique dans plusieurs sphères de leur vie. Les trajectoires susceptibles d'expliquer leur risque accru de victimisation et les implications pour l'intervention sont discutées. Les besoins multiples de ces jeunes soulignent l'importance de développer une vision plus holistique de leur vécu de victimisation et de leurs problèmes. Une plus grande collaboration des divers intervenants et organismes impliqués auprès des jeunes est susceptible de favoriser une approche moins fragmentée et une intervention mieux adaptée.

MOTS-CLÉS - Protection de la jeunesse, victimisation, polyvictimisation, victimisation multiple.

1. Université de Montréal, Pavillon Lionel-Groulx, École de service social, C. P. 6128, Succursale Centre-ville, Montréal (Québec), Canada, H3C 3J7. 


\section{Introduction}

Au Québec, 77244 signalements ont été traités par les services de protection de la jeunesse (PJ) en 2011-2012 et de ce nombre, 32661 ont été retenus pour évaluation (Association des centres jeunesse du Québec [ACJQ], 2013). Selon l'étude canadienne sur l'incidence des signalements de cas de violence et de négligence envers les enfants (Trocmé et al., 2010), on estime que les organismes canadiens de PJ ont fait enquête sur 235842 cas en 2008, la maltraitance ayant été confirmée pour 85440 cas (36\%). La situation pourrait être chronique pour près de la moitié de ces enfants qui avaient déjà été l'objet d'une enquête dans le passé. En outre, plus d'une forme de maltraitance était présente dans $18 \%$ des situations confirmées par les enquêtes (Trocmé et al., 2010). Bien que ces données soient préoccupantes, elles ne révèlent que les formes de victimisation les plus sérieuses (Finkelhor \& Ormrod, 2001; Bowlus et al, 2003) et uniquement une minorité des jeunes maltraités font l'objet d'une enquête par les services de PJ (MacMillan et al., 2003; Sedlack et al., 2010).

Ces données ne nous renseignent pas sur les autres formes de violence que peuvent vivre les enfants négligés ou maltraités alors qu'un grand nombre de jeunes sont exposés à la violence dans de multiples contextes (Green et al., 2000; Menard \& Huizinga, 2001; Finkelhor et al., 2005; Mrug et al., 2008). En fait, les jeunes victimes de violence au sein de la famille sont aussi fréquemment la cible de victimisation extrafamiliale (Duncan, 1999; Perry et al., 2001; Shields \& Cicchetti, 2001; Baldry, 2003; Cyr et al., 2012). Le terme polyvictimisation a ainsi été introduit pour désigner ces jeunes qui accumulent un nombre de victimisations distinctes supérieur à la moyenne au cours d'une période donnée (Finkelhor et al., 2007), incluant l'intimidation et la violence des pairs, la victimisation sexuelle, les crimes contre la propriété, l'exposition à la violence dans la famille et la communauté ainsi que la maltraitance et la négligence. Plusieurs enfants exposés à de multiples événements traumatiques de nature interpersonnelle vivent des séquelles désignées par le terme trauma complexe (Herman, 1992; van der Kolk, 2005), lequel inclut des troubles de l'attachement, des difficultés de gestion des émotions et des comportements, des troubles de dissociation et de concept de soi (Cook et al., 2005). Ces séquelles sont fréquemment rapportées chez les jeunes pris en charge en PJ (Trocmé et al., 2010) qui sont plus nombreux à avoir recours à des 
services de santé mentale comparativement aux jeunes de la population générale (Farmer et al., 2001), soulignant que plusieurs ont pu être exposés de manière chronique à de multiples formes de violence.

Le fait de vivre dans une famille dangereuse (caractérisée par la violence au sein de la famille), dans une famille à problèmes multiples (ex.: problèmes de consommation, stress financier, chômage, etc.) ou de souffrir de troubles de santé mentale sont trois trajectoires menant à la polyvictimisation chez les jeunes (Finkelhor et al., 2007). Au Canada, on sait que les jeunes pris en charge par la PJ sont nombreux à vivre avec un parent victime $(46 \%)$ ou auteur $(13 \%)$ de violence familiale et ayant des problèmes multiples tels l'alcoolisme (21\%), la toxicomanie (17\%) et le manque de soutien social (39\%) (Trocmé et al., 2010), ce qui pourrait les placer à risque de polyvictimisation.

Cette étude vise à documenter et à comparer la polyvictimisation et les différentes formes de victimisation vécues par les jeunes pris en charge par la Direction de la protection de la jeunesse (DPJ) au Québec avec celles vécues par les jeunes de la population générale. Examiner les formes de victimisation qui touchent de manière disproportionnée les jeunes pris en charge est susceptible de favoriser l'identification des jeunes à risque et de fournir des pistes d'intervention plus adéquates et concertées. Considérant le manque de services et de ressources pour les jeunes pris en charge par les organismes de protection de l'enfance (Glisson, 1996; Burns et al., 2004; Hurlburt et al., 2004), révéler l'ampleur de leurs expériences traumatiques est également susceptible de favoriser le financement de services et de politiques publiques visant à leur venir en aide.

\section{Méthodologie}

\section{Procédure de collecte des données}

L'échantillon comprend des participants issus de trois enquêtes téléphoniques, l'une auprès de jeunes dans les services de PJ et les deux autres (2-11 ans et 12-17 ans) auprès d'échantillons représentatifs de la population. Ces enquêtes ont été effectuées avec le Juvenile Victimization Questionnaire, soit le même questionnaire qui est employé pour les enquêtes téléphoniques aux États-Unis. Pour les enfants âgés de 2 à 11 ans, l'entrevue était effectuée auprès du responsable connaissant le mieux le vécu quotidien de l'enfant. Pour les adolescents de 12 à 17 ans, 
elle était réalisée directement auprès de l'adolescent concerné. Les entrevues étaient d'une durée de 20 à 30 minutes.

La première enquête a été réalisée auprès d'un échantillon de 220 jeunes (2-17 ans) pris en charge par trois centres jeunesse du Québec. La sélection de l'échantillon a été effectuée en plusieurs étapes. Les centres jeunesse ont fourni des listes aléatoires de clients recevant des services à l'application des mesures, indiquant que leur sécurité et leur développement étaient compromis selon la Loi sur la protection de la jeunesse. Dans les deux centres jeunesse de Montréal, l'intervenant responsable du dossier excluait ensuite certains participants de la liste, s'il jugeait que ces derniers pouvaient être traumatisés par une enquête au sujet de leurs expériences de victimisation. S'il n'y avait pas de contre-indication clinique, l'intervenant responsable demandait le consentement des parents et des jeunes âgés de 12 et plus afin que leurs coordonnées soient fournies à l'équipe de recherche. Dans le cas du centre jeunesse de Québec, un intervenant a été dégagé pour la recherche et il se chargeait d'étudier les dossiers, d'exclure les participants pour lesquels des contre-indications cliniques étaient indiquées dans le dossier informatisé et de contacter les parents et les jeunes pour obtenir leur consentement. Une fois les coordonnées obtenues, une lettre était envoyée aux participants potentiels qui étaient par la suite contactés par téléphone. Encore une fois, le consentement de tous les parents ainsi que des jeunes de 12 ans et plus était obtenu avant l'entrevue téléphonique. Les données sociodémographiques étaient obtenues en interrogeant brièvement le parent lorsque possible. Le participant était invité à passer l'entrevue dans une pièce où il ne pouvait être entendu par une tierce personne et était avisé que l'interviewer contacterait la DPJ si les informations révélées indiquaient que le développement ou la sécurité d'un jeune était possiblement compromis. Les répondants recevaient un chèque-cadeau afin de les remercier de leur participation.

Les deux autres enquêtes ont été réalisées auprès de la population du Québec par une firme spécialisée. Les bases de sondage employées pour ces enquêtes étaient à génération aléatoire de numéros de téléphone (GANT). Une première enquête a été réalisée auprès de 1400 adolescents de 12 à 17 ans et une deuxième auprès de 1401 parents d'enfants âgés de 2 à 11 ans. Pour ces deux enquêtes, si plus d'un enfant vivait dans le ménage, un seul était sélectionné aléatoirement. Le consentement verbal de tous les répondants et des parents des jeunes de 12 et 13 ans 
a été obtenu avant l'entretien. Afin de garantir l'anonymat des répondants, les entretiens ont été effectués selon une procédure d'aveuglement volontaire, garantissant ainsi que les numéros de téléphone des répondants demeurent inconnus des interviewers. Compte tenu de cette procédure, les participants n'ont reçu aucune compensation et ne pouvaient être signalés à la DPJ. Cependant, les participants étaient référés à une ligne d'aide dans le cas des jeunes et à des ressources pour ce qui est des parents à la fin de l'entretien. Les procédures pour les trois enquêtes ont été approuvées par le comité d'éthique des institutions d'appartenance des chercheurs et des centres jeunesse concernés.

\section{Description de l'échantillon}

L'échantillon comprend 220 participants recrutés en centre jeunesse et 2801 participants de la population générale du Québec, et il inclut approximativement le même pourcentage de garçons et de filles. Ce sont 1538 adolescents de 12 ans et plus qui ont eux-mêmes fourni des informations concernant leur victimisation alors que pour $49 \%$ de l'échantillon, c'est la personne connaissant le mieux le vécu quotidien de l'enfant de moins de 12 ans qui a été interrogée. Comme l'illustre le tableau 1 , l'échantillon populationnel diffère de l'échantillon recruté en centre jeunesse quant à plusieurs variables sociodémographiques, reflétant ainsi qu'il s'agit de populations fort distinctes.

Les jeunes de la population générale sont significativement plus nombreux à vivre avec deux parents dans les deux groupes d'âge. La scolarité des parents diffère significativement selon l'échantillon dans les deux groupes d'âge. Alors que la majorité de l'échantillon populationnel rapporte un niveau post-secondaire, les parents des jeunes pris en charge sont significativement plus nombreux à rapporter un diplôme d'études secondaires ou à ne pas l'avoir obtenu. Chez les moins de 12 ans, c'est la moitié des parents d'enfants pris en charge qui n'ont pas obtenu leur diplôme d'études secondaires (contre seulement $3 \%$ dans la population générale). Nous comparons ainsi des populations dont les caractéristiques sociodémographiques diffèrent fortement, certaines (niveau d'éducation des parents, monoparentalité souvent liée à un revenu moindre) étant associées à des risques de victimisation plus élevés, laissant dès lors envisager plus de victimisation chez les jeunes pris en charge étant donné leur plus grande précarité sociale. 
TABLEA U 1

Caractéristiques de l'échantillon

\begin{tabular}{|c|c|c|c|c|c|c|c|c|c|c|c|c|}
\hline \multirow{3}{*}{$\begin{array}{l}\hat{\text { Age }} \\
\text { Sexe }\end{array}$} & \multicolumn{6}{|c|}{ 2-11 ans } & \multicolumn{6}{|c|}{$12-17$ ans } \\
\hline & \multicolumn{2}{|c|}{$\begin{array}{l}\text { Centres jeunesse } \\
\quad(n=82)\end{array}$} & \multicolumn{2}{|c|}{$\begin{array}{l}\text { Population } \\
(n=1401)\end{array}$} & \multicolumn{2}{|c|}{$\begin{array}{c}\text { Total } \\
(n=1483)\end{array}$} & \multicolumn{2}{|c|}{$\begin{array}{l}\text { Centres jeunesse } \\
\qquad(n=138)\end{array}$} & \multicolumn{2}{|c|}{$\begin{array}{l}\text { Population } \\
(n=1400)\end{array}$} & \multicolumn{2}{|c|}{$\begin{array}{c}\text { Total } \\
(\mathrm{n}=1538)\end{array}$} \\
\hline & $\mathbf{n}$ & $\%$ & $\mathbf{n}$ & $\%$ & $\mathbf{n}$ & $\%$ & $\mathbf{n}$ & $\%$ & $\mathbf{n}$ & $\%$ & $\mathbf{n}$ & $\%$ \\
\hline Filles & 39 & 48,0 & 686 & 49,0 & 725 & 49,0 & 74 & 54,0 & 704 & 50,0 & 778 & 51,0 \\
\hline Garçons & 43 & 52,0 & 715 & 51,0 & 758 & 51,0 & 64 & 46,0 & 696 & 50,0 & 760 & 49,0 \\
\hline \multicolumn{13}{|l|}{ Milieu de vie } \\
\hline Deux parents ${ }^{\mathrm{a}}$ & 9 & 11,0 & 1246 & $89,0 * * *$ & 1255 & 85,0 & 15 & 11,0 & 896 & $64,0 * * *$ & 911 & 59,0 \\
\hline Mère monoparentale & 35 & $43,0 * * *$ & 86 & 6,0 & 121 & 8,0 & 30 & 22,0 & 268 & 19,0 & 298 & 19,0 \\
\hline Père monoparental & 10 & $12,0 * * *$ & 10 & 1,0 & 20 & 1,0 & 4 & 3,0 & 67 & 5,0 & 71 & 5,0 \\
\hline Garde partagée & - & - & 42 & 3,0 & 42 & 3,0 & 2 & 1,0 & 151 & $11,0 * * *$ & 153 & 10,0 \\
\hline Famille d'accueil/adoptive & 19 & $23,0 * * *$ & 15 & 1,0 & 34 & 2,0 & 30 & $22,0 * * *$ & 4 & 0,3 & 34 & 2,0 \\
\hline Centre d'accueil & 2 & $2,0 * *$ & - & - & 2 & 0,1 & 41 & $30,0 * * *$ & - & - & 41 & 3,0 \\
\hline Autre $^{b}$ & 7 & $9,0 * * *$ & 2 & 0,1 & 9 & 0,6 & 16 & $12,0 * * *$ & 14 & 1,0 & 30 & 2,0 \\
\hline
\end{tabular}


TABLEAU 1 (suite)

\begin{tabular}{|c|c|c|c|c|c|c|c|c|c|c|c|c|}
\hline \multirow[t]{3}{*}{ Âge } & \multicolumn{6}{|c|}{$2-11$ ans } & \multicolumn{6}{|c|}{$12-17$ ans } \\
\hline & \multicolumn{2}{|c|}{$\begin{array}{l}\text { Centres jeunesse } \\
\quad(n=82)\end{array}$} & \multicolumn{2}{|c|}{$\begin{array}{l}\text { Population } \\
(n=1401)\end{array}$} & \multicolumn{2}{|c|}{$\begin{array}{c}\text { Total } \\
(n=1483)\end{array}$} & \multicolumn{2}{|c|}{$\begin{array}{l}\text { Centres jeunesse } \\
\quad(n=138)\end{array}$} & \multicolumn{2}{|c|}{$\begin{array}{l}\text { Population } \\
(n=1400)\end{array}$} & \multicolumn{2}{|c|}{$\begin{array}{c}\text { Total } \\
(\mathrm{n}=1538)\end{array}$} \\
\hline & $\mathbf{n}$ & $\%$ & $\mathbf{n}$ & $\%$ & $\mathbf{n}$ & $\%$ & $\mathrm{n}$ & $\%$ & $\mathbf{n}$ & $\%$ & $\mathbf{n}$ & $\%$ \\
\hline \multicolumn{13}{|l|}{ Scolaritéc } \\
\hline Aucun diplôme & 41 & $50,0^{* * *}$ & 36 & 3,0 & 77 & 5,0 & 31 & $23,0 * * *$ & 36 & 3,0 & 67 & 4,0 \\
\hline $\begin{array}{l}\text { Études secondaires ou } \\
\text { équivalent }\end{array}$ & 28 & 34,0 ** & 303 & 22,0 & 331 & 22,0 & 64 & $46,0 * * *$ & 244 & 17,0 & 308 & 20,0 \\
\hline Études post-secondaires & 13 & 16,0 & 1059 & $76,0^{* * *}$ & 1072 & 72,0 & 26 & 19,0 & 945 & $68,0^{* * *}$ & 971 & 63,0 \\
\hline Refus/NSP & - & & 2 & 0,1 & 2 & 0,1 & 17 & 12,0 & 175 & 13,0 & 192 & 13,0 \\
\hline
\end{tabular}

Note: La somme des pourcentages n'est pas toujours de $100 \%$ puisque les valeurs ont été arrondies à deux décimales.

${ }^{a}$ Incluant les jeunes vivant avec deux parents biologiques ou un parent biologique et son conjoint.

b "Autre» comprend les jeunes vivant avec un grand-parent, un frère ou une sœur adulte, un autre membre de la parenté, des amis adultes.

c Personne ayant le plus haut niveau de scolarité atteint dans le milieu de vie de l'enfant.

*** Différence significative, $\mathrm{p}<, 001$ (tests $x^{2}$ ).

** Différence significative, $\mathrm{p}<, 01$ (tests $x^{2}$ ). 


\section{Instruments}

Le Juvenile Victimization Questionnaire (Hamby \& Finkelhor, 2004) permet de décrire 33 formes de victimisation avec ou sans violence pouvant avoir été vécues directement et indirectement par les adolescents au cours de la dernière année. La version pour les plus jeunes est la même, mais ne décrit que 32 formes de victimisation (la violence dans les relations amoureuses ne s'appliquant pas). Cette version a été administrée au responsable connaissant le mieux le vécu quotidien de l'enfant de moins de 12 ans. Les données démographiques recueillies incluaient l'âge et le sexe de l'enfant, l'origine ethnique de l'enfant et de ses parents, et la structure familiale.

\section{Méthode d'analyse}

Compte tenu que la source des données diffère selon l'âge de l'enfant (parent pour les moins de 12 ans et jeune lui-même), les prévalences et les comparaisons entre la population et les jeunes pris en charge sont présentées de manière distincte. Une mesure du nombre total de victimisations différentes survenues dans la dernière année a été créée afin d'identifier les jeunes étant plus fortement victimisés. La mesure de la polyvictimisation est évaluée à partir du nombre de victimisations différentes vécues lors d'événements distincts. La mesure exclut ainsi la victimisation répétée: un enfant ayant été victime à répétition de la même forme de victimisation sera considéré comme ayant vécu une seule forme de victimisation. De plus, seules les victimisations vécues au cours d'événements distincts sont considérées, afin de ne pas surestimer le nombre de victimisations vécues. Cette mesure continue permet de déterminer le nombre moyen de victimisations vécues et permet d'identifier les jeunes plus fortement victimisés.

\section{Résultats}

Vols et méfaits

Tel que présenté au tableau 2, les jeunes pris en charge ( $45 \%$ chez les moins de 12 ans et $73 \%$ chez les $12-17$ ans) sont significativement plus nombreux à avoir été la cible de cette forme de victimisation ${ }^{2}$ dans la

2. Incluant le vol qualifié, le vol simple et le vandalisme ou les méfaits sur la propriété. 
TABLEA U 2

Prévalence des victimes de vols et méfaits, sur une période d'une année

\begin{tabular}{|c|c|c|c|c|c|c|c|c|c|c|c|c|}
\hline \multirow[t]{3}{*}{ Âge } & \multicolumn{6}{|c|}{ 2-11 ans } & \multicolumn{6}{|c|}{$12-17$ ans } \\
\hline & \multicolumn{2}{|c|}{$\begin{array}{l}\text { Centres jeunesse } \\
\qquad(n=82)\end{array}$} & \multicolumn{2}{|c|}{$\begin{array}{l}\text { Population } \\
(n=1 \text { 401) }\end{array}$} & \multicolumn{2}{|c|}{$\begin{array}{c}\text { Total } \\
(n=1 \text { 483) }\end{array}$} & \multicolumn{2}{|c|}{$\begin{array}{l}\text { Centres jeunesse } \\
\quad(n=138)\end{array}$} & \multicolumn{2}{|c|}{$\begin{array}{l}\text { Population } \\
(n=1400)\end{array}$} & \multicolumn{2}{|c|}{$\begin{array}{c}\text { Total } \\
(n=1538)\end{array}$} \\
\hline & $\mathbf{n}$ & $\%$ & $\mathbf{n}$ & $\%$ & $\mathbf{n}$ & $\%$ & $\mathbf{n}$ & $\%$ & $\mathbf{n}$ & $\%$ & $\mathbf{n}$ & $\%$ \\
\hline Victimes $^{\mathrm{a}}$ & 37 & $45,0 * * *$ & 304 & 22,0 & 341 & 23,0 & 100 & $73,0 * * *$ & 396 & 28,0 & 496 & 32,0 \\
\hline Vol qualifié & 1 & 1,0 & 27 & 2,0 & 28 & 2,0 & 16 & $12,0 * * *$ & 38 & 3,0 & 54 & 4,0 \\
\hline Vol simple & 14 & $17,0^{* *}$ & 86 & 6,0 & 100 & 7,0 & 81 & $59,0 * * *$ & 259 & 19,0 & 340 & 22,0 \\
\hline Vandalisme/méfaits & 24 & 29,0 ** & 219 & 16,0 & 243 & 16,0 & 42 & $30,0 * * *$ & 155 & 11,0 & 197 & 13,0 \\
\hline
\end{tabular}

a «Victimes» inclut les jeunes ayant vécu au moins une victimisation de cette catégorie.

*** Différence significative, $\mathrm{p}<, 001$ (tests $x^{2}$ ).

** Différence significative, $p<, 01$ (tests $x^{2}$ ). 
dernière année comparativement aux jeunes de la population générale ( $22 \%$ chez les moins de 12 ans et $28 \%$ chez les $12-17$ ans). Chez les enfants, la forme la plus souvent vécue est le vandalisme d'un objet appartenant à l'enfant (16\% de l'échantillon total), suivi du vol simple (7\% de l'échantillon total). Les jeunes pris en charge sont significativement plus nombreux à rapporter ces formes de violence: plus d'un enfant sur quatre $(29 \%)$ a été victime de vandalisme et près d'un sur cinq (17\%) a été victime de vol. Chez les adolescents, la prévalence des trois formes de victimisation de la catégorie vols et méfaits diffère significativement selon le groupe, la forme la plus fréquente étant le vol simple. Pratiquement trois fois plus d'adolescents suivis par un centre jeunesse ont été victimes de vandalisme $(30 \%$ versus $11 \%$ dans la population) et quatre fois plus ont été la cible de vol qualifié $(12 \%$ contre $3 \%$ dans la population).

\section{Voies de fait, harcèlement et intimidation}

Les voies de fait, le harcèlement et l'intimidation font partie d'une catégorie de victimisation ${ }^{3}$ très commune chez les jeunes $(37 \%$ de l'échantillon total) et sont la forme la plus souvent vécue par les enfants de moins de 12 ans (40\%). Encore une fois, les jeunes pris en charge sont significativement plus nombreux que la population générale $(65 \%$ contre $39 \%$ chez les $2-11$ ans et $75 \%$ contre $31 \%$ chez les $12-17$ ans) à rapporter avoir vécu au moins une forme de victimisation de cette catégorie dans les douze mois précédant l'enquête (Tableau 3). L'agression de la part d'un pair ou de la fratrie est la forme la plus commune chez les jeunes de moins de 12 ans, étant vécue par plus de la moitié des enfants pris en charge $(54 \%)$ et près d'un tiers $(30 \%)$ des enfants de la population. D'autres formes assez fréquentes dans ce groupe d'âge sont l'intimidation verbale (19\% de l'échantillon total) et les voies de fait simples (14\% de l'échantillon total), aussi significativement plus fréquentes dans le groupe d'enfants pris en charge. D'autres formes, bien que moins prévalentes, sont aussi significativement plus souvent vécues par les enfants du groupe centre jeunesse. Ils sont trois

3. Incluant les voies de fait simples, les voies de fait armées, les tentatives de voies de fait, les enlèvements ou tentatives d'enlèvement, les agressions motivées par la haine, les agressions de groupe ou de gangs, les agressions de la part de la fratrie, les voies de fait aux parties intimes, le caïdage, l'intimidation verbale et la violence dans les relations amoureuses. 
TA B LE A U 3

Prévalence des victimes de voies de fait, harcèlement et intimidation, sur une période d'une année

\begin{tabular}{|c|c|c|c|c|c|c|c|c|c|c|c|c|}
\hline \multirow{3}{*}{ Âge } & \multicolumn{6}{|c|}{$2-11$ ans } & \multicolumn{6}{|c|}{$12-17$ ans } \\
\hline & \multicolumn{2}{|c|}{$\begin{array}{c}\text { Centres jeunesse } \\
(n=82)\end{array}$} & \multicolumn{2}{|c|}{$\begin{array}{l}\text { Population } \\
(\mathrm{n}=1 \mathrm{401})\end{array}$} & \multicolumn{2}{|c|}{$\begin{array}{c}\text { Total } \\
(n=1483)\end{array}$} & \multicolumn{2}{|c|}{$\begin{array}{c}\text { Centres jeunesse } \\
(n=138)\end{array}$} & \multicolumn{2}{|c|}{$\begin{array}{l}\text { Population } \\
(n=1400)\end{array}$} & \multicolumn{2}{|c|}{$\begin{array}{c}\text { Total } \\
(n=1538)\end{array}$} \\
\hline & $\mathbf{n}$ & $\%$ & $\mathbf{n}$ & $\%$ & $\mathbf{n}$ & $\%$ & $\mathbf{n}$ & $\%$ & $\mathbf{n}$ & $\%$ & $\mathbf{n}$ & $\%$ \\
\hline Victimes de voies de fait $^{\text {a }}$ & 53 & $65,0 * * *$ & 543 & 39,0 & 596 & 40,0 & 104 & $75,0 * * *$ & 431 & 31,0 & 535 & 35,0 \\
\hline Voies de fait armées & 7 & $9,0^{*}$ & 42 & 3,0 & 49 & 3,0 & 18 & $13,0 * * *$ & 44 & 3,0 & 62 & 4,0 \\
\hline Voies de fait simples & 31 & $38,0 * * *$ & 182 & 13,0 & 213 & 14,0 & 63 & $46,0 * * *$ & 207 & 15,0 & 270 & 18,0 \\
\hline Tentative de voies de fait & 13 & $16,0 * * *$ & 55 & 4,0 & 68 & 5,0 & 39 & $28,0 * * *$ & 55 & 4,0 & 94 & 6,0 \\
\hline Enlèvement/tentative & 0 & - & 4 & 0,3 & 4 & 0,3 & 4 & $3,0 * *$ & 5 & 0,4 & 9 & 0,6 \\
\hline Agression haineuse & 3 & $4,0^{*}$ & 7 & 0,5 & 10 & 0,7 & 16 & $12,0^{* * *}$ & 22 & 2,0 & 38 & 3,0 \\
\hline $\begin{array}{l}\text { Agression par un groupe/ } \\
\text { gang }\end{array}$ & 4 & $5,0^{*}$ & 17 & 1,0 & 21 & 1,0 & 20 & $15,0 * * *$ & 35 & 3,0 & 55 & 4,0 \\
\hline $\begin{array}{l}\text { Agression d'un pair } \\
\text { ou fratrie }\end{array}$ & 44 & 54,0 ** & 413 & 30,0 & 457 & 31,0 & 61 & $44,0 * * *$ & 209 & 15,0 & 270 & 18,0 \\
\hline $\begin{array}{l}\text { Voies de fait aux parties } \\
\text { intimes (non sexuel) }\end{array}$ & 1 & 1,0 & 42 & 3,0 & 43 & 3,0 & 15 & 11,0 ** & 65 & 5,0 & 80 & 5,0 \\
\hline Caïdage & 13 & 16,0 & 143 & 10,0 & 156 & 11,0 & 20 & $15,0 * * *$ & 43 & 3,0 & 63 & 4,0 \\
\hline Intimidation verbale & 25 & 31,0 ** & 258 & 18,0 & 283 & 19,0 & 50 & $36,0 * * *$ & 173 & 12,0 & 223 & 15,0 \\
\hline $\begin{array}{l}\text { Violence physique dans les } \\
\text { relations amoureuses }\end{array}$ & $\mathrm{N} / \mathrm{A}$ & - & $\mathrm{N} / \mathrm{A}$ & - & $\mathrm{N} / \mathrm{A}$ & - & 24 & $17,0 * * *$ & 37 & 3,0 & 61 & 4,0 \\
\hline
\end{tabular}

a «Victimes de voies de fait» inclut les jeunes ayant vécu au moins une victimisation de cette catégorie, excluant le caïdage et l'intimidation verbale.

*** Différence significative, $p<, 001$ (tests $x^{2}$ ).

** Différence significative, $\mathrm{p}<, 01$ (tests $\left.x^{2}\right)$

* Différence significative, $p<, 05$ (tests $x^{2}$ ). 
fois plus nombreux à avoir été agressés avec un objet ou une arme, quatre fois plus nombreux à avoir été la cible d'une tentative de voies de fait, cinq fois plus nombreux à avoir été agressés par un groupe de jeunes et huit fois plus nombreux à avoir été victimes d'une agression motivée par la haine.

Chez les adolescents (12-17 ans), toutes les formes de victimisation de cette catégorie sont significativement plus souvent rapportées par les jeunes pris en charge que par ceux de la population générale, les formes les plus courantes étant les voies de fait simples ( $46 \%$ pour le groupe centre jeunesse et $15 \%$ dans la population) et l'agression de la part d'un pair ou de la fratrie (44\% groupe centre jeunesse versus $15 \%$ dans la population). Les agressions haineuses sont six fois plus fréquentes chez les adolescents pris en charge, alors que l'agression par un groupe de jeunes ou un gang, ainsi que l'intimidation verbale, le sont cinq fois plus. La violence dans les relations amoureuses est rapportée par près d'un adolescent pris en charge sur cinq ( $17 \%$ versus $3 \%$ dans la population) et même la forme de victimisation la moins fréquente, l'enlèvement, est sept fois plus commune ( $3 \%$ versus $0,4 \%$ ) chez les adolescents recrutés via les centres jeunesse.

\section{Maltraitance}

La différence entre le groupe de jeunes pris en charge et le groupe de la population concernant la maltraitance ${ }^{4}$ est frappante $(34 \%$ versus $5 \%$ chez les moins de 12 ans et $42 \%$ versus $9 \%$ chez les $12-17$ ans, voir tableau 4), les jeunes de moins de 12 ans étant pratiquement sept fois plus nombreux que ceux de la population à avoir vécu une forme de maltraitance durant l'année à l'étude. L'abus psychologique est la forme la plus fréquente, vécue par une proportion significativement plus grande de jeunes pris en charge dans les deux groupes d'âge. Les abus physiques, vécus par $3 \%$ des adolescents et $1 \%$ des jeunes de moins de 12 ans, ont été subis par 18 fois plus d'adolescents et par 30 fois plus d'enfants pris en charge. Alors que moins de $1 \%$ des enfants de la population ont été négligés, un enfant de moins de 12 ans sur dix suivi par un centre jeunesse a subi cette forme de victimisation. Puisque la prise en charge s'effectue dans le but de protéger les enfants de l'abus

4. Incluant l'abus physique, l'abus psychologique, la négligence et les conflits de garde/l'enlèvement par un parent. 
TA B LE A U 4

Prévalence des victimes de maltraitance, sur une période d'une année

\begin{tabular}{|c|c|c|c|c|c|c|c|c|c|c|c|c|}
\hline \multirow[t]{3}{*}{ Âge } & \multicolumn{6}{|c|}{$2-11$ ans } & \multicolumn{6}{|c|}{$12-17$ ans } \\
\hline & \multicolumn{2}{|c|}{$\begin{array}{l}\text { Centres jeunesse } \\
\quad(n=82)\end{array}$} & \multicolumn{2}{|c|}{$\begin{array}{l}\text { Population } \\
(n=1401)\end{array}$} & \multicolumn{2}{|c|}{$\begin{array}{c}\text { Total } \\
(n=1483)\end{array}$} & \multicolumn{2}{|c|}{$\begin{array}{l}\text { Centres jeunesse } \\
\quad(n=138)\end{array}$} & \multicolumn{2}{|c|}{$\begin{array}{l}\text { Population } \\
(n=1400)\end{array}$} & \multicolumn{2}{|c|}{$\begin{array}{c}\text { Total } \\
(n=1538)\end{array}$} \\
\hline & $\mathbf{n}$ & $\%$ & $\mathbf{n}$ & $\%$ & $\mathbf{n}$ & $\%$ & n & $\%$ & $\mathbf{n}$ & $\%$ & $\mathbf{n}$ & $\%$ \\
\hline Victimes & 28 & $34,0 * * *$ & 68 & 5,0 & 96 & 7,0 & 58 & $42,0 * * *$ & 126 & 9,0 & 184 & 12,0 \\
\hline Abus physique & 12 & $15,0 * * *$ & 7 & 0,5 & 19 & 1,0 & 25 & $18,0 * * *$ & 20 & 1,0 & 45 & 3,0 \\
\hline $\begin{array}{l}\text { Abus émotionnel/ } \\
\text { psychologique }\end{array}$ & 17 & $21,0 * * *$ & 55 & 4,0 & 72 & 5,0 & 50 & $36,0 * * *$ & 111 & 8,0 & 161 & 11,0 \\
\hline Négligence & 8 & $10,0 * * *$ & 7 & 0,5 & 15 & 1,0 & 6 & $4,0^{* *}$ & 8 & 0,6 & 14 & 1,0 \\
\hline $\begin{array}{l}\text { Conflit de garde/ } \\
\text { enlèvement par un parent }\end{array}$ & 7 & $9,0 * * *$ & 3 & 0,2 & 10 & 0,7 & 4 & $3,0^{* *}$ & 3 & 0,2 & 7 & 0,5 \\
\hline
\end{tabular}

a «Victimes» inclut les jeunes ayant vécu au moins une victimisation de cette catégorie.

*** Différence significative, $p<, 001$ (tests $x^{2}$ ).

** Différence significative, $p<, 01$ (tests $x^{2}$ ). 
T A B LE A U 5

Prévalence de la victimisaton sexuelle sur une période d'une année

\begin{tabular}{|c|c|c|c|c|c|c|c|c|c|c|c|c|}
\hline \multirow[t]{3}{*}{ Âge } & \multicolumn{6}{|c|}{$2-11$ ans } & \multicolumn{6}{|c|}{$12-17$ ans } \\
\hline & \multicolumn{2}{|c|}{$\begin{array}{l}\text { Centres jeunesse } \\
\qquad(n=82)\end{array}$} & \multicolumn{2}{|c|}{$\begin{array}{l}\text { Population } \\
(n=1401)\end{array}$} & \multicolumn{2}{|c|}{$\begin{array}{c}\text { Total } \\
(n=1483)\end{array}$} & \multicolumn{2}{|c|}{$\begin{array}{l}\text { Centres jeunesse } \\
\qquad(n=138)\end{array}$} & \multicolumn{2}{|c|}{$\begin{array}{l}\text { Population } \\
(\mathrm{n}=1400)\end{array}$} & \multicolumn{2}{|c|}{$\begin{array}{c}\text { Total } \\
(\mathrm{n}=1538)\end{array}$} \\
\hline & $\mathbf{n}$ & $\%$ & $\mathbf{n}$ & $\%$ & $\mathbf{n}$ & $\%$ & $\mathbf{n}$ & $\%$ & $\mathbf{n}$ & $\%$ & $\mathbf{n}$ & $\%$ \\
\hline Victimisation sexuelle ${ }^{a, b}$ & 7 & 9,0 & 34 & 2,0 & 41 & 3,0 & 39 & $28,0 * * *$ & 115 & 8,0 & 154 & 10,0 \\
\hline Agression sexuelle $^{b, c}$ & 2 & 2,0 & 4 & 0,3 & 6 & 0,4 & 17 & $12,0^{* * *}$ & 28 & 2,0 & 45 & 3,0 \\
\hline $\begin{array}{l}\text { Agression sexuelle, adulte } \\
\text { connu }^{d}\end{array}$ & 0 & - & 0 & - & 0 & - & 3 & 2,0 & 3 & 0,2 & 6 & 0,4 \\
\hline $\begin{array}{l}\text { Agression sexuelle, } \\
\text { étranger }^{d}\end{array}$ & 0 & - & 0 & - & 0 & - & 4 & 3,0 & 6 & 0,4 & 10 & 0,7 \\
\hline Agression sexuelle, pairs ${ }^{b, d}$ & 2 & 2,0 & 3 & 0,2 & 5 & 0,3 & 5 & 4,0 & 4 & 0,3 & 9 & 0,6 \\
\hline Viol ou tentative & 0 & - & 2 & 0,1 & 2 & 0,1 & 12 & $9,0 * * *$ & 20 & 1,0 & 32 & 2,0 \\
\hline $\begin{array}{l}\text { Exposition sexuelle } \\
\text { (exhibitionnisme) }^{\mathrm{b}}\end{array}$ & 5 & 6,0 & 11 & 0,8 & 16 & 1,0 & 13 & $9,0^{* *}$ & 40 & 3,0 & 53 & 3,0 \\
\hline Harcèlement sexuel $^{\mathrm{b}}$ & 2 & 2,0 & 22 & 2,0 & 24 & 2,0 & 21 & $15,0 * * *$ & 45 & 3,0 & 66 & 4,0 \\
\hline
\end{tabular}

a «Victimisation sexuelle» inclut les jeunes ayant vécu au moins une victimisation de cette catégorie.

b Différences entre les groupes chez les 2-11 ans non calculées car $n$ est inférieur à 10.

c «Agression sexuelle» inclut les jeunes ayant vécu au moins une victimisation de cette catégorie, excluant l'exposition sexuelle et le harcèlement sexuel.

${ }^{d}$ Différences entre les groupes chez les 12-17 ans non calculées car $n$ est inférieur à 10.

*** Différence significative, $\mathrm{p}<.001$. (tests $x^{2}$ ).

** Différence significative, $p<, 01$ (tests $x^{2}$ ).

* Différence significative, $p<, 05$ (tests $x^{2}$ ). 
de leurs parents, il est normal que la prévalence soit plus élevée dans ce groupe.

\section{Victimisation sexuelle}

Bien que la victimisation sexuelle $e^{5}$ soit la catégorie de victimisation la moins prévalente dans l'échantillon, elle touche plus les jeunes pris en charge, soit près d'un adolescent sur trois $(28 \%$, comparativement à $8 \%$ dans la population) et près d'un enfant de moins de 12 ans sur dix $(9 \%$, comparativement à $2 \%$ dans la population). Chez les adolescents, toutes les formes de victimisation de cette catégorie touchent davantage les jeunes recrutés à travers les centres jeunesse. La forme la plus courante est le harcèlement sexuel (3\% de l'échantillon total), dont la prévalence est cinq fois plus grande parmi les adolescents suivis par la PJ (15\% versus $3 \%$ chez les adolescents de la population générale). Chez les enfants de moins de 12 ans, bien que les jeunes pris en charge semblent plus touchés par les victimisations sexuelles les plus sévères, les prévalences sont faibles et ne permettent pas de vérifier avec fiabilité les différences statistiques entre les groupes.

\section{Témoin de violence}

L'exposition à la violence est une autre catégorie de violence ${ }^{6}$ fréquemment vécue par les jeunes, touchant un quart d'entre eux. Puisque les adolescents sont plus souvent hors du milieu familial sans supervision, ils sont aussi plus nombreux à vivre cette forme de victimisation (39\%) que les plus jeunes (12\%). Être témoin de voies de fait simples est la forme de victimisation la plus couramment rapportée (6\% des moins de 12 ans et $28 \%$ des $12-17$ ans); elle est significativement plus prévalente dans le groupe recevant des services de la PJ. Les autres formes les plus courantes diffèrent selon l'âge. Les plus jeunes sont plutôt témoins de violence dans le milieu familial, soit de violence conjugale et d'abus physiques envers la fratrie, phénomènes qui touchent significativement plus les enfants pris en charge $(16 \%$ versus $0,4 \%$ pour la

5. Incluant l'agression sexuelle par un adulte connu, par un étranger, par un pair, le viol ou tentative de viol, l'exposition sexuelle et le harcèlement sexuel.

6. Incluant le fait d'être témoin de violence conjugale, d'abus physiques sur la fratrie, de voies de fait armées et simples, de meurtre, d'avoir eu son domicile cambriolé, d'avoir eu un proche assassiné, d'avoir été exposé à des émeutes, tirs ou bombes, ou à une guerre. 
TABLEA U 6

Prévalence des victimes témoins de violence, sur une période d'une année

\begin{tabular}{|c|c|c|c|c|c|c|c|c|c|c|c|c|}
\hline \multirow[t]{3}{*}{ Âge } & \multicolumn{6}{|c|}{$2-11$ ans } & \multicolumn{6}{|c|}{$12-17$ ans } \\
\hline & \multicolumn{2}{|c|}{$\begin{array}{c}\text { Centres jeunesse } \\
(n=82)\end{array}$} & \multicolumn{2}{|c|}{$\begin{array}{l}\text { Population } \\
(\mathrm{n}=1401)\end{array}$} & \multicolumn{2}{|c|}{$\begin{array}{c}\text { Total } \\
(n=1483)\end{array}$} & \multicolumn{2}{|c|}{$\begin{array}{c}\text { Centres jeunesse } \\
(n=138)\end{array}$} & \multicolumn{2}{|c|}{$\begin{array}{l}\text { Population } \\
(n=1400)\end{array}$} & \multicolumn{2}{|c|}{$\begin{array}{c}\text { Total } \\
(n=1538)\end{array}$} \\
\hline & $\mathrm{n}$ & $\%$ & $\mathrm{n}$ & $\%$ & $n$ & $\%$ & $\mathrm{n}$ & $\%$ & $\mathrm{n}$ & $\%$ & $\mathrm{n}$ & $\%$ \\
\hline Témoin de violence $^{a}$ & 30 & $37,0 * * *$ & 145 & 10,0 & 175 & 12,0 & 115 & $83^{* * *}$ & 478 & 34,0 & 593 & 39,0 \\
\hline $\begin{array}{l}\text { Témoin de violence } \\
\text { conjugale }\end{array}$ & 13 & $16,0 * * *$ & 6 & 0,4 & 19 & 1,0 & 6 & $4^{* *}$ & 10 & 0,7 & 16 & 1,0 \\
\hline $\begin{array}{l}\text { Témoin d'abus physiques } \\
\text { sur la fratrie }\end{array}$ & 4 & 5,0 ** & 4 & 0,3 & 8 & 0,5 & 13 & $9 * * *$ & 5 & 0,4 & 18 & 1,0 \\
\hline $\begin{array}{l}\text { Témoin de voies de fait } \\
\text { armées }\end{array}$ & 2 & 2,0 & 23 & 2,0 & 25 & 2,0 & 52 & $38^{* * *}$ & 137 & 10,0 & 189 & 12,0 \\
\hline $\begin{array}{l}\text { Témoin de voies de fait } \\
\text { simples }\end{array}$ & 18 & $22,0 * * *$ & 67 & 5,0 & 85 & 6,0 & 94 & $68^{* * *}$ & 343 & 25,0 & 437 & 28,0 \\
\hline Cambriolage du domicile & 4 & 5,0 & 52 & 4,0 & 56 & 4,0 & 22 & $16^{* * *}$ & 76 & 5,0 & 98 & 6,0 \\
\hline Proche assassiné & 2 & $2,0^{*}$ & 4 & 0,3 & 6 & 0,4 & 20 & $15^{* * *}$ & 17 & 1,0 & 37 & 2,0 \\
\hline Témoin de meurtre & 0 & - & 0 & - & 0 & - & 8 & $6 * * *$ & 3 & 0,2 & 11 & 0,7 \\
\hline $\begin{array}{l}\text { Exposition à des tirs, } \\
\text { bombes, émeutes }\end{array}$ & 2 & $2,0^{*}$ & 4 & 0,3 & 6 & 0,4 & 30 & $22 * * *$ & 45 & 3,0 & 75 & 5,0 \\
\hline Exposition à la guerre & 0 & - & 0 & - & 0 & - & 0 & - & 6 & 0,4 & 6 & 0,4 \\
\hline
\end{tabular}

a Témoin de violence inclut les jeunes ayant vécu au moins une victimisation de cette catégorie.

$* * *$ Différence significative, $\mathrm{p}<, 001$ (tests $x^{2}$ ).

** Différence significative, $p<, 01$ (tests $x^{2}$ ).

* Différence significative, $p<, 05$ (tests $x^{2}$ ). 
violence conjugale et $5 \%$ versus $0,3 \%$ pour la violence envers la fratrie). Chez les adolescents, plus d'un jeune sur dix $(12 \%)$ a été témoin d'une agression armée, la prévalence étant pratiquement quatre fois plus élevée parmi les adolescents pris en charge (38\% versus $10 \%$ dans la population). L'ampleur de la violence vécue par les adolescents du groupe centre jeunesse apparaît encore plus marquée en observant les différences pour des événements particulièrement violents et généralement moins prévalents, tels être témoin de meurtre $(6 \%$ contre $0,2 \%$ dans la population), être exposé à des tirs ou des émeutes ( $22 \%$ contre $3 \%$ ) ou avoir un proche ayant été assassiné (15\% versus $1 \%)$.

\section{Polyvictimisation}

Le nombre de violences différentes (excluant la répétition de la même forme) accumulées par un même jeune au cours de la dernière année est rapporté dans le tableau 7. On remarque que la majorité des jeunes ont vécu au moins une forme de violence. Chez les enfants de moins de 12 ans, pratiquement six enfants de la population sur dix ont vécu de la violence ( $59 \%$ ) alors que c'est le cas de plus de huit enfants sur dix dans le groupe suivi par la DPJ (82\%). Parmi les enfants victimes, le nombre moyen de formes de violence est de 3,5 dans le groupe pris en charge et de 1,9 parmi les victimes de la population. Bien qu'il n'y ait pas de différence significative selon la prise en charge quant à la proportion d'enfants (2-11 ans) ayant vécu d'une à trois formes de violence, la différence est marquée lorsqu'il s'agit de quatre formes et plus (34\% parmi les enfants pris en charge contre 5,1\% dans la population). Chez les plus jeunes, la norme est de subir entre une et trois formes de violence au cours d'une année; cependant, plus d'un tiers des enfants recevant des services de la DPJ ont vécu plus de trois formes annuellement.

Chez les adolescents, pratiquement sept jeunes de la population sur dix $(66 \%)$ ont vécu de la violence cours de l'année alors que c'est le cas pour pratiquement tous les adolescents pris en charge (96\%). Pour les adolescents, la norme est également de subir entre un et trois types de violence annuellement (51\%) alors que chez ceux recevant des services d'un centre jeunesse, la norme est d'en vivre au moins quatre formes $(66 \%)$; plus du tiers $(36 \%)$ ayant vécu au moins sept formes de violence distinctes. En fait, le nombre moyen de violences vécues parmi les victimes est pratiquement trois fois plus élevé chez 
TABLEA U 7

Prévalence de la polyvictimisation, sur une période d'une année

\begin{tabular}{|c|c|c|c|c|}
\hline \multirow[t]{2}{*}{ Âge } & \multicolumn{2}{|c|}{$2-11$ ans } & \multicolumn{2}{|c|}{$12-17$ ans } \\
\hline & $\begin{array}{l}\text { Centres jeunesse } \\
\quad(n=82)\end{array}$ & $\begin{array}{l}\text { Population } \\
(n=1401)\end{array}$ & $\begin{array}{l}\text { Centres jeunesse } \\
\quad(n=138)\end{array}$ & $\begin{array}{l}\text { Population } \\
(n=1400)\end{array}$ \\
\hline & $\%$ & $\%$ & $\%$ & $\%$ \\
\hline Aucune victimisation & 18,0 & $41,0 * * *$ & 4,0 & $34,0 * * *$ \\
\hline $1-3$ victimisations & 48,0 & 54,0 & 30,0 & $51,0 * * *$ \\
\hline $4-6$ victimisations & $27,0 * * *$ & 5,0 & $30,0 * * *$ & 10,0 \\
\hline 7 victimisations et plus & $7,0 * * *$ & 0,1 & $36,0 * * *$ & 2,0 \\
\hline Nombre moyen de victimisations $\mathrm{s}^{\mathrm{a}}$ & 3,5 & 1,9 & 6 & 2,4 \\
\hline
\end{tabular}

*** Différence significative, $\mathrm{p}<, 001$ (tests $\left.x^{2}\right)$.

** Différence significative, $p<, 01$ (tests $x^{2}$ ).

* Différence significative, $\mathrm{p}<, 05$ (tests $x^{2}$ ).

a Le nombre moyen de victimisations est calculé parmi les victimes et exclut les jeunes non victimisés

2-11 ans: $n=67$ pour les jeunes de l'échantillon centres jeunesse; $n=824$ pour les jeunes de l'échantillon populationnel

12-17 ans: $n=132$ pour les jeunes de l'échantillon centres jeunesse; $n=885$ pour les jeunes de l'échantillon populationnel. 
les adolescents pris en charge (6) que chez les adolescents de la population générale $(2,4)$.

\section{Discussion}

Au Québec, plusieurs jeunes vivent un nombre élevé de violences au cours d'une année, et l'étude révèle une prévalence nettement plus élevée de polyvictimisation parmi ceux pris en charge que parmi ceux de la population générale. Les jeunes pris en charge par les organismes de PJ au Québec semblent donc vivre une situation de victimisation plus chronique. D'une part, ils sont touchés dans plusieurs domaines de leur vie, ce qui peut être nuisible à leur développement. D'autre part, ils font face à de multiples facteurs de risque qui correspondent aux quatre trajectoires de polyvictimisation documentées dans la littérature (Finkelhor et al., 2007; Finkelhor, 2008). En effet, la première trajectoire, celle des familles dangereuses, peut expliquer le vécu de plusieurs jeunes pris en charge par la DPJ. Elle représente une famille dans laquelle la violence est commune, incluant la maltraitance et la violence intrafamiliale (Finkelhor, 2008). Les enfants sont ainsi exposés à plusieurs victimisations dans leur milieu familial, ce qui a des conséquences sur leur développement, et les met ensuite à risque de polyvictimisation dans la sphère extrafamiliale (Finkelhor, 2008). La deuxième trajectoire, celle des familles aux problèmes multiples, mettrait les jeunes à risque à cause du manque de supervision, de l'exposition accrue à des agresseurs potentiels et de la négligence émotionnelle qui sont souvent associés aux familles aux prises avec de multiples problèmes (toxicomanie, maladies, itinérance, etc.) (Finkelhor et al., 2007; Finkelhor, 2008). Dans cette trajectoire, la négligence émotionnelle que vivent les jeunes, associée à leur manque de protection, feraient d'eux des proies faciles et augmenteraient ainsi leur risque de revictimisation. La troisième trajectoire identifiée par Finkelhor et ses collègues (2007) correspond à vivre dans une communauté à risque ou un voisinage dangereux, soit une région caractérisée par une grande concentration de criminalité, communément désignée comme une zone chaude (bot spots) en criminologie (Farrel \& Sousa, 2001). Dans ce type de voisinage, les risques d'exposition à la violence sont accrus et les jeunes qui y vivent sont exposés à plus d'agresseurs potentiels, ce qui augmente leur risque de subir de la violence, même si leur milieu familial est adéquat (Finkelhor, 2008). La dernière trajectoire est en lien avec les 
caractéristiques personnelles des jeunes, soit leur tempérament, certains déficits, troubles émotionnels, ou problèmes d'adaptation qui feraient d'eux des cibles faciles aux yeux des agresseurs (Finkelhor, 2008). Bien que la détresse psychologique n'ait pas été évaluée dans cette étude, il a été démontré que les symptômes de dépression, de colère et d'anxiété sont des prédictifs de toutes les formes de victimisation (Cuevas et al., 2010). Il est possible que ces jeunes soient stigmatisés et par conséquent plus souvent victimisés par leurs pairs, ou encore que leur capacité à se protéger et à reconnaître les situations potentiellement dangereuses soit réduite (Cuevas et al., 2009). Similairement, leurs problèmes peuvent accroître leur risque de maltraitance et de négligence de la part de leurs parents puisqu'ils peuvent créer des exigences supplémentaires pour ces derniers (Finkelhor, 2008). Cette trajectoire est particulièrement inquiétante en regard de la victimisation chronique que vivent les jeunes pris en charge qui ont des niveaux de besoins élevés (Trocmé et al., 2010), soulignant leurs nombreux problèmes d'adaptation. Les conséquences psychologiques engendrées par leur victimisation pourraient ainsi les placer à risque de victimisations subséquentes (Cuevas et al., 2010). Il a aussi été démontré dans d'autres études que la polyvictimisation est également plus prédictive des troubles d'adaptation que les victimisations considérées individuellement (Finkelhor et al., 2007). Cela signifie que plusieurs jeunes sont possiblement enfermés dans un cercle vicieux en étant chroniquement victimes, ce qui accroît tant leur risque de développer des troubles adaptatifs que d'êtres re-victimisés.

\section{Limites}

L'étude comporte certaines limites. Mentionnons tout d'abord que nos données proviennent d'une seule source (l'adolescent ou le parent selon le cas) et n'ont donc pas été corroborées par une autre source de données. Il est donc possible que certains parents ou jeunes aient pu minimiser ou exagérer les victimisations vécues. L'accès aux jeunes pris en charge par la protection de la jeunesse pour des fins de recherche était difficile et plusieurs sentinelles ont dû fournir leur consentement avant que le jeune puisse être invité à participer à l'étude et à fournir son propre consentement. Dans un premier temps, plusieurs jeunes pris en charge ont été exclus par les intervenants responsables pour des motifs cliniques, des jeunes possiblement fortement polyvictimisés. De plus, comme l'accord des parents était nécessaire pour ce groupe, il y a 
lieu de s'interroger concernant les jeunes exclus parce que leurs parents s'opposaient à leur participation. Il est possible que ces derniers étaient victimes de maltraitance intrafamiliale, mais le refus des parents pouvait aussi simplement être le fait d'une méfiance de leur part, puisqu'ils étaient informés que toute information révélant un risque potentiel pour la sécurité et le développement d'un enfant serait signalée à la DPJ. Bien que cela n'était pas le cas pour l'échantillon populationnel, puisque la procédure d'aveuglement volontaire ne permettait pas d'identifier les répondants, le consentement des parents était requis pour la participation des adolescents de 12 et 13 ans, et nous ignorons les caractéristiques des jeunes ainsi exclus. En fait, les caractéristiques de l'ensemble des jeunes exclus ou ayant refusé eux-mêmes de participer à l'étude sont inconnues.

L'échantillon recruté en centre jeunesse est aussi majoritairement composé d'adolescents car peu de parents de jeunes de 2 à 11 ans ont accepté de prendre part à l'étude. Encore une fois, nous ignorons si c'est la présence de victimisation intrafamiliale ou une méfiance face au système de prise en charge qui a motivé leur refus. Ainsi, malgré les efforts de l'équipe afin de recruter un échantillon représentatif des jeunes pris en charge par les services de protection de la jeunesse au Québec, nous ne pouvons affirmer que l'échantillon était représentatif de l'ensemble de ces derniers et il est fort possible que les cas plus lourds aient été exclus par les intervenants. Similairement, l'échantillon populationnel est composé majoritairement de familles intactes avec des parents au niveau d'éducation élevé et n'est donc pas représentatif de l'ensemble de la population du Québec, ce qui limite la généralisation des données.

Dans un même ordre d'idées, il importe de rappeler qu'il y a des différences sociodémographiques importantes entre les jeunes de la population et ceux suivis par la protection de la jeunesse dans les échantillons comparés. Ces différences sont susceptibles d'être en lien avec la prévalence plus élevée de violence et de polyvictimisation chez les jeunes pris en charge et il importe d'interpréter les résultats avec prudence.

Par ailleurs, malgré le souci d'effectuer l'entrevue avec le parent connaissant le mieux le vécu quotidien des enfants de moins de 12 ans, il est possible que ce dernier n'ait pas été au courant de toutes les victimisations vécues et ait été réticent à rapporter certaines formes (parce qu'il était lui-même agresseur, ou encore par honte ou désirabilité 
sociale). Les études américaines ont interrogé directement les jeunes dès l'âge de dix ans et n'ont dénoté aucune différence significative entre les taux de victimisation rapportés par les parents de jeunes de 8 et 9 ans et ceux rapportés par les jeunes de 10-11ans (Finkelhor et al., 2005). Cependant, pour des raisons éthiques, il a été impossible d'interroger les jeunes directement avant l'âge de douze ans au Québec.

\section{Conclusion}

À notre connaissance, la présente étude est la seule qui compare la polyvictimisation des jeunes de la population générale et celle des jeunes pris en charge par les services de PJ. Puisque la majorité des jeunes pris en charge pour maltraitance vivent d'autres victimisations en concomitance et font face à de la victimisation chronique, il y a lieu de s'interroger sur l'intervention de manière sectorielle. Il importe de développer des mécanismes permettant de connaître les diverses formes de victimisation vécues par les jeunes dans diverses sphères de leur vie afin d'identifier ceux qui sont polyvictimisés puisqu'ils sont non seulement plus nombreux à afficher des troubles d'adaptation sévères, mais aussi plus à risque de re-victimisation. En effet, une vision générale des problématiques vécues est susceptible de favoriser une approche moins fragmentée et une plus grande collaboration des divers intervenants et organismes pouvant venir en aide aux jeunes. En ce sens, un défi pour les intervenants est de développer une vision plus holistique des divers problèmes vécus par la clientèle leur permettant de déterminer et d'accéder aux ressources et expertises qui pourront les soutenir dans l'intervention (Cyr et al., 2012). Les jeunes pris en charge qui vivent de la violence dans plusieurs contextes ont des besoins multiples et un risque accru de victimisation en raison de leurs symptômes de détresse psychologique. Il importe ainsi de favoriser l'accessibilité à des soins et services spécialisés pour les traumatismes complexes. La collaboration entre les services de PJ, les acteurs du milieu scolaire, des services communautaires, des services sociaux et des soins de santé apparaît donc essentielle pour éviter de morceler le vécu des jeunes pris en charge (Cyr et al., 2012). Puisqu'il est possible que la situation de polyvictimisation de ces jeunes soit associée à une ou plusieurs des trajectoires identifiées par Finkelhor (2008), une exploration des facteurs familiaux, des caractéristiques des jeunes et de leur environnement est susceptible de fournir des pistes d'intervention auprès des polyvic- 
times suivies par la DPJ. Les programmes d'aide sociale gouvernementaux peuvent également contribuer à réduire la situation précaire des familles des jeunes pris en charge.

\section{Références}

Association des Centres Jeunesse du Québec (2013). Bilan des directeurs de la protection de la jeunesse /Directeurs provinciaux 2012. Montréal: Association des Centres Jeunesse du Québec.

Baldry, A. C. (2003). Bullying in schools and exposure to domestic violence. Child Abuse and Neglect, 27 (7), 713-732.

Bowlus, A., McKenna, K., Day, T., \& Wright, D. (2003). The Economic Costs and Consequences of Child Abuse in Canada. Report to the Law Commission of Canada: 172 pages.

Burns, B. J., Phillips, S. D., Wagner, H. R., Barth, R. P., Kolko, D. J., Campbell, Y., \& Landsverk, J. (2004). Mental Health Need and Access to Mental Health Services by Youths Involved with Child Welfare: A National Survey. Journal of the American Academy of Child and Adolescent Psychiatry, 43 (8), 960970.

Cook, A., Spinazzola, J., Ford, J., Lanktree, C., Blaustein, M., Cloitre, M., DeRosa, R., Hubbard, R., Kagan, R., Liautaud, J., Mallah, K., Olafson, E., \& Van der Kolk, B. (2005). Complex Trauma in Children and Adolescents. Psychiatric Annals, 35, 390-398.

Cuevas, C. A., Finkelhor, D., Clifford, C., Ormrod, R. K., \& Turner, H. A. (2010). Psychological distress as a risk factor for re-victimization in children. Child Abuse $\mathcal{O}$ Neglect, 34 (4), 235-243.

Cuevas, C. A., Finkelhor, D., Ormrod, R. K., \& Turner, H. A. (2009). Psychiatric Diagnosis as a Risk Marker for Victimization in a National Sample of Children. Journal of Interpersonal Violence, 24 (4), 636-652.

Cyr, K., Chamberland, C., Lessard, G., Clément, M-Ė., Collin-Vézina, D., Wemmers, J.-A., Gagné, M.-H., \& Damant, D. (2012). Polyvictimization in a clinical sample of children and youths. Psychology of Violence, 2 (4), 385400.

Duncan, R. D. (1999). Maltreatment by parents and peers: The relationship between child abuse, bully victimization, and psychological distress. Child Maltreatment, 4 (1), 45-55.

Eckenrode, J., Laird, M., \& Doris, J. (1993). School performance and disciplinary problems among abused and neglected children. Developmental Psycho$\log y, 29(1), 53-62$.

Farmer, E. M. Z., Burns, B. J., Chapman, M., Phillips, S. D., Angold, A., \& Costello, E. J. (2001). Use of mental health services by youth in contact with social services. Social Service Review, 75, 605-624.

Farrel, G., \& Sousa, W. (2001). Preventing Repeat Victimization. In M. Tonry \& D. Farrington (eds.). Building a Safer Society: Strategic Approaches to crime 
Prevention, Crime and Justice: A Review of Research (vol. 19), (469-534). Chicago, IL: University of Chicago.

Finkelhor, D. (2008). Childhood Victimization Violence, Crime, and Abuse in the Lives of Young People. New York, NY: Oxford University Press.

Finkelhor, D., Hamby, S. L., Ormrod, R. K., \& Turner, H. A. (2005). The Juvenile Victimization Questionnaire: Reliability, validity, and national norms. Child Abuse er Neglect, 29 (4), 383-412.

Finkelhor, D. \& Ormrod, R. K. (2001). Offenders Incarcerated for Crimes Against Juveniles. Juvenile Justice Bulletin - NCJ191028 (1-12).

Finkelhor, D., Ormrod, R. K., \& Turner, H. A. (2007). Re-Victimization patterns in a national longitudinal sample of children and youth. Child Abuse $\sim$ Neglect, 31 (5), 479-502.

Glisson, C. (1996). Judicial and service decisions for children entering state custody: The limited role of mental health. Social Service Review, 70 (2), 257-281.

Green, B. L., Goodman, L. A., Krupnick, J. L., Corcoran, C. B., Petty, R. M., Stockton, P., \& Stern, N. M. (2000). Outcomes of single versus multiple trauma exposure in a screening sample. Journal of Traumatic Stress, 13 (2), 271-286.

Hamby, S. L., \& Finkelhor, D. (2004). The Comprehensive Juvenile Victimization Questionnaire. Durham, NH: University of New Hampshire.

Herman, J. L. (1992). Complex PTSD: a syndrome in survivors of prolonged and repeated Trauma. Journal of Traumatic Stress, 5 (3), 377-391.

Hulburt, M. S., Leslie, L. K., Landsverk, J., Barth, R. P., Burns, B. J., Gibbons, R. D., Slymen, D. J., \& Zhang, J. J. (2004). Contextual predictors of mental health service use among children open to child welfare. Archives of General Psychiatry, 61 (12), 1217-1224.

MacMillan, H. L., Jamieson, E., \& Walsh, C. A. (2003). Reported contact with child protection services among those reporting child physical and sexual abuse: Results from a community survey. Child Abuse $\mathfrak{W}$ Neglect, 27 (12), 1397-1408.

Menard, S., \& Huizinga, D. (2001). Repeat victimization in a high-risk neighborhood sample of adolescents. Youth $\mathfrak{F}$ Society, 32 (4), 447-472.

Mrug, S., Loosier, P. S., \& Windle, M. (2008). Violence exposure across multiple contexts: Individual and joint effects on adjustment. American Journal of Orthopsychiatry, 78 (1), 70-84.

Perry, D. G., Hodges, E. V. E., \& Eagan, S. K. (2001). Determinants of chronic victimization by peers: A review and new model of family influence. In J. Juvonen \& S. Graham (eds.), Peer Harassment in school: The plight of the vulnerable and victimized (73-104). New York, NY: Guilford Press.

Sedlak, A., Mettenburg, J., Basena, M. Petta, I., McPherson, K., Green, A., \& $\mathrm{Li}, \mathrm{S}$. (2010). The fourth national incidence study of child abuse and neglect (NIS-4): Report to Congress, executive Summary. Washington, DC: U.S. Department of Health and Human Services, Administration for Children and Families. 
Shields, A., \& Cicchetti, D. (2001). Parental maltreatment and emotion dysregulation as risk factors for bullying and victimization in middle childhood. Journal of Clinical Child Psychology, 30 (3), 349-363.

Trocmé, N., Fallon, B., MacLaurin, B., Sinha, V., Black, T., Fast, E., Felstiner, C., Hélie, S., Turcotte, D., Weightman, P., Douglas, J., \& Holroyd, J., (2010) Étude canadienne sur l'incidence des signalements de cas de violence et de négligence envers les enfants - 2008. Ottawa: Agence de Santé Publique du Canada.

Van der Kolk, B. (2005). Developmental Trauma Disorder. Toward a rational diagnosis for children with complex trauma histories. Psychiatric Annals, 35, 401-408.

ABSTRACT - This article compares the annual violence experiences of a sample of Quebec's children (2 to 17 years old) followed by child welfare agencies, with those of a sample of children from the general population. The results reveals that the children followed by child welfare agencies experienced significantly more violence and that many are chronically victimized in many areas of their lives. The authors discuss the possible trajectories linked to their increased victimization risk and the implications for intervention in child welfare. Given the multiple needs and victimizations experiences of these children, a more holistic approach and more collaboration between agencies and other professionals involved with children is recommended.

KEY WORDS - Child welfare, victimization, polyvictimization, multiple victimization.

RESUMEN - El presente artículo compara la violencia vivida anualmente por una muestra de menores quebequenses (de 2 a 17 años) puestos bajo la tutela de la dirección de la protección de la juventud (DPJ). Los resultados demuestran que los menores institucionalizados viven significativamente más violencia y que una gran parte de ellos se enfrentan a una violencia crónica en varias esferas de sus vidas. Las trayectorias susceptibles de explicar el riesgo aumentado de victimización y las implicaciones para la intervención serán tratadas en este artículo. Las múltiples necesidades de estos jóvenes subrayan la importancia de desarrollar una visión más holística de sus vivencias de victimización y de sus problemas. Una mayor colaboración de los diversos actores y organismos implicados con los jóvenes es susceptible de favorizar un enfoque menos fragmentado y una intervención más adaptada.

PALABRAS CLAVE - Protección del menor, victimización, poli-victimización, victimización múltiple. 